\title{
Dual technique of reconfiguration and capacitor placement for distribution system
}

\author{
Haider Fathi Kadom ${ }^{1}$, Ali Nasser Hussain ${ }^{2}$, Waleed Khalid Shakir Al-Jubori ${ }^{3}$ \\ ${ }^{1,2}$ Department of Electrical Power Engineering Techniques, Middle Technical University, Iraq \\ ${ }^{3}$ Department of Electrical Power Engineering Techniques, AL-Furat AL-Awsat Technical University, Iraq
}

\begin{abstract}
Article Info
Article history:

Received Apr 8, 2019

Revised Aug 26, 2019

Accepted Aug 30, 2019

Keywords:

DSR

Losses cost reduction

MBBO

$\mathrm{OCP}$

Voltage profile

ABSTRACT

Radial Distribution System (RDS) suffer from high real power losses and lower bus voltages. Distribution System Reconfiguration (DSR) and Optimal Capacitor Placement (OCP) techniques are ones of the most economic and efficient approaches for loss reduction and voltage profile improvement while satisfy RDS constraints. The advantages of these two approaches can be concentrated using of both techniques together. In this study two techniques are used in different ways. First, the DSR technique is applied individually. Second, the dual technique has been adopted of DSR followed by OCP in order to identify the technique that provides the most effective performance. Three optimization algorithms have been used to obtain the optimal design in individual and dual technique. Two IEEE case studies (33bus, and 69 bus) used to check the effectiveness of proposed approaches. A Direct Backward Forward Sweep Method (DBFSM) has been used in order to calculate the total losses and voltage of each bus. Results show the capability of the proposed dual technique using Modified Biogeography Based Optimization (MBBO) algorithm to find the optimal solution for significant loss reduction and voltage profile enhancement. In addition, comparisons with literature works done to show the superiority of proposed algorithms in both techniques.
\end{abstract}

Copyright $(0) 2020$ Institute of Advanced Engineering and Science. All rights reserved.

\section{Corresponding Author:}

Ali Nasser Hussain,

Department of Electrical Power Engineering Techniques,

Middle Technical University,

Muasker Al Rashid Street, 7F7P+JG Baghdad, Baghdad, Al Zafranyia, Iraq.

Email: alinasser1974@yahoo.com

\section{INTRODUCTION}

The loss reduction on distribution network is the main goal in efficient operation. From both control and protection sides, distribution system is frequently being in radial topology. Due to low voltage level, high flowing current and high $\mathrm{R} / \mathrm{X}$ ratio in the RDS, the biggest portion of losses is in distribution system comparing to the transmission and generation systems losses. The techniques proposed for losses reduction are OCP and DSR to improve the system behavior and augment loss reduction taking into account the economic aspect. DSR is an efficient method to solve this problem by search to the most efficient configuration [1]. DSR first proposed by Merlin and Back using mixture of optimized and heuristic algorithms to find the optimal operating structure of RDS with an objective of loss reduction [2]. There are different methods were used for optimal reconfiguration. Generally, DSR is essential to provide interest to as many consumers as possible during a fault and maintenance purposes. It also reduces the real power losses and provides the balancing for loads in order to prevent overloading and overheating of network lines. 
A huge care has been done recently on the DSR with an objective of real power loss reduction. It studied with different objective functions, constraints, optimization algorithms and distribution system topologies in references [3-8]. These studies not study the DSR with a multi objective function of real power losses reduction and voltage profile improvements without considering precense of Distributed Generation (DG) and Photo Voltaic (PV).

OCP technique first proposed by Baran and $\mathrm{Wu}$, which is a reactive power injection process requires installing the shunt capacitors on the RDS. Therefore, the optimal locations and sizes of capacitors have been considered as an optimization problem [9]. Many of previous studies have been done for OCP problem using multiple objective functions and different intelligent algorithms as in references [10-14]. Some of these studies suffer from bus voltage and capacitor size constraints were not achieved. In addition, the location of each capacitor was based on Loss Sensitivity Index (LSI) in these studies, which may take more time and force the optimized algorithm to select these locations.

DSR alone or OCP alone cannot confirm a loss reduction and voltage enhancement of buses significantly; therefore, a new approach has employed to combine both techniques for getting better performance in RDS. Many studies found for combining both techniques sequentially, firstly reconfiguration processes and then capacitor placement (DSR-OCP) as done in references [15-17]. These works concentrated on real power losses with annual losses cost reduction and neglecting voltage improvement aspect. Furthermore, a few works in the literature that applied the dual technique for a large standard system such as 69 bus RDS.

In this paper, two techniques are used, DSR as an individual technique and dual technique of DSROCP with three modified versions of optimization algorithms Modified Biogeography Based Optimization (MBBO), Binary Teaching Learning Based Optimization (BTLBO) and Discrete Dolphin Echolocation (DDE). These modified algorithms have been used for the first time in the individual and dual techniques in order to enhance the quality searching of the original versions and to prevent a slide into the premature convergence to local minima. The optimal solution contains the optimal selection of tie switches, sizes and locations of capacitors based on multi-objective functions and RDS constraints. Two IEEE standard networks are used 33 bus and 69 bus to check the effectiveness of proposed algorithms. The whole design of the proposed work with three-optimization algorithms has compared with the available literature works for the same objective functions and constraints to test the efficiency and validity of proposed work. The results confirmed the efficiency of these modified versions over their original versions in terms of increasing the losses reduction and voltage profile improvement. Also, the proposed work confirmed the RDS constraints and not considered DG or PV implementation. Finally, the MBBO algorithm is one of best and modern efficient evolutionary algorithms for optimized problems due to high accuracy and lower computation requirements.

\section{RESEARCH METHOD}

DSR technique is a good approach to reduce power loss and maintain the voltage profile within permissible values. Furthermore, loss can be minimized extremely by OCP technique so consecutive usage of both techniques can further reduce losses and improve voltage profile than using them separately. Two applications are used here, first one DSR individually approach, and the second is dual DSR-OCP approach.

\subsection{Load flow and objective functions}

A Direct Backward Forward Sweep Method (DBFSM) load flow used here to calculate bus voltage, branch current and total losses [18]. Conventional load flow methods of transmission systems are not suitable for RDS because of high R/X ratio, unbalanced loading, and radial structure. In addition, the tolerance value is specified equals $1 \times 10^{-6}$. Multi objective functions are used to obtain the lower real power losses, and voltage enhancing for the buses using proposed techniques and optimization algorithms. These objective functions (obj.f.) are:

- Real power losses reduction:

$$
\begin{aligned}
& \text { obj.f. } 1=P_{\text {loss }} \\
& P_{\text {loss }}=\sum_{l=1}^{N_{b r}} P_{\text {lossl }} \mathrm{KW} \\
& P_{\text {lossl }}=I_{l}^{2} * R_{l} \mathrm{KW}
\end{aligned}
$$

Where: $P_{\text {loss }}$ : Total real power losses.

$N_{b r}:$ Number of branches.

$R_{l}$ : Resistance of branch $l$. 
$I_{l} \quad$ : Current flow in branch $l$.

- Voltage profile improvement (obj.f. 2), here voltage must be within acceptable limits.

$$
\text { obj.f. } 2=V_{C} * R e_{v}+C_{C} * R e_{i}
$$

Where: $V_{C}$ : Bus voltage limits.

$C_{C}$ : Branch current limits.

$R e_{v}$ : Retribution factor for bus voltage. This factor equals zero if the bus voltage within acceptable limits.

$R e_{i}$ : Retribution factor for branch current. This equals zero if branch current not exceed the thermal limit.

Therefore, the final objective function (obj.f. ) will be combination of above individual ones as:

$$
\text { obj.f. }=\text { obj.f. } 1+o b j . f .2
$$

\subsection{Constraints}

The constraints that confirm the superior performance of the RDS are classified to technical and operational constraints as follow:

\subsubsection{Technical constraints}

These constraints are defined as an inequality limits and divided into:

a. Voltage consraints

Voltage value for each bus must be within their acceptable domain to sustain power quality as:

$$
\left|V_{\text {jmin }}\right| \leq\left|V_{j}\right| \leq\left|V_{\text {jmax }}\right|, \mathrm{j} \in N_{b}, N_{b} \text { : number of buses }
$$

Then, the standard minimum and maximum voltages are 0.95 p.u and 1.05 p.u respectively [19].

b. Current constraints

Branch current must not exceed its limited value from protection side and investigate power supply continuity

$$
\left|I_{l}\right| \leq\left|I_{l m a x}\right|, l \in N_{b r}, N_{b r}: \text { is number of branches }
$$

Where the maximum branch capacity is specified in [20].

c. Capacitors size constraint

The total capacitor size $\left(Q_{C T}\right)$ inserted must not override the total reactive power load $\left(Q_{\text {load }}\right)$ of the RDS as:

$$
Q_{C T} \leq Q_{\text {load }}
$$

\subsubsection{Operational constraints}

These constraints are defined as an equality limits and divided into:

a. Radial constraints and all loads are covered given in [8]

This condition can confirmed by determining the determinant of bus incidence matrix [A] as

b. Power balance constraint

$$
P_{\text {Sup }}=P_{\text {Dem }}+P_{\text {loss }}
$$

Where: $P_{\text {Sup }}$ is total supplied power, $P_{\text {Dem }}$ is total load power.

\subsection{Optimization algorithms}

Three optimization algorithms are used in this work, MBBO, BTLBO and DDE for both individual and dual techniques. These algorithms are described in detail respectively.

\subsubsection{MBBO Algorithm}

The BBO algorithm first introduced by Dan Simon in 2008 inspired from nature of geological distribution for living beings [21]. The original algorithm suffers from trapping in local optima and weakly 
behavior [22]. The MBBO algorithm introduced in this paper to solve these drawbacks, also it first time used for DSR and OCP problems and demonstrated promising results over their original version in terms of robustness and good performance.

The steps of MBBO are as follow:

1. Define algorithm parameters as explain in Table 1.

2. Calculate Keep number (K. NO.) of population as:

$$
\text { K. NO. =Keep rate } * \text { Pop. NO. }
$$

Where, Pop. NO. is a Population number.

3. Calculate Habitat number (Hab. NO.) as:

$$
\text { Hab. NO. = Pop. NO. - K. NO. }
$$

4. Calculate Emigration rate $(E M)$ and then Immigration rate $(I M)$ by:

$$
\begin{aligned}
& E M=\frac{1}{\text { Pop.NO.-1 }} \\
& I M=1-E M
\end{aligned}
$$

5. Calculate emigration probability $\left(P_{E M}\right)$ using $I M$ values as:

$$
P_{E M}=E M \quad \text { if rand } \leq I M
$$

Where, rand is random numbers between $0 \& 1$; Then, final value of $P_{E M}$ is determine by [22]:

$$
P_{E M}(\infty)=\frac{P_{E M S}}{\sum_{S=1}^{P o p . N O \cdot} P_{E M S}}
$$

Where, $S$ is specified species number.

6. Determine updated Suitability Index $\left(S I_{n}^{k+1}\right)$ for nth solution from previous nth and mth solution [23]:

$$
S I_{n}^{k+1}=S I_{n}^{k}+\beta *\left(S I_{m}^{k}-S I_{n}^{k}\right)
$$

Where, $\beta$ is a variable range between $0 \& 1$.

7. Check mutation probability value with rand value, if it bigger than or equal to rand value then,

$$
S I_{n}^{k+1}=S I_{n}^{k+1}+E * \text { rand }
$$

Where, $E$ is Elite number.

8. Repeat steps (2-7) until max iterations achieved.

9. End.

Table 1. Optimal values of MBBO algorithm

\begin{tabular}{lc}
\hline Parameter & Value \\
\hline Pop. $\boldsymbol{N O}$. & 30 \\
Mutation probability & 0.1 \\
$\boldsymbol{E}$ & 2 \\
Maximum number of iterations & 50 (33 bus) \\
(RDS) & $100(69$ bus $)$ \\
maximum $I M$ & 1 \\
maximum $E M$ & 1 \\
Keep rate & 0.2 \\
$\boldsymbol{\beta}$ & 0.9 \\
\hline
\end{tabular}

\subsubsection{BTLBO algorithm}

The TLBO algorithm is an intelligent Meta heuristic type introduced by Rao et al. in 2012 which consists from two processes, teaching process and learning process [24]. The population number is equal to number of students while the number of variables is equal to the lectures introduced to students. In the present work because of discrete nature of DSR and OCP techniques, then the binary code for this 
algorithm has been used at first time to solve the prposed individual and dual techniques. Furthermore, the original version of this algorithm suffer from lower performance because of the differ nature of the applied algorithm with the proposed techniques such that easily slip in the local optimum solution. The steps of BTLBO Algorithm are:

1. Initializing algorithm parameters as shown in Table 2.

2. Teaching process, this stage begin by specifying Teacher Factor value $\left(T . F_{v a l}\right)$ and as given by reference [25]:

$$
T \cdot F_{\text {val }}=\operatorname{round}(1+\text { rand })
$$

Where, round is an integer number, then $T . F_{v a l}$ in the range is (1-2).

3. Determine the Average Difference (Avg. Diff.) if the average degree of lecture $(u)$ for the student $(z)$ at iteration $(k)$ is enhanced from old value $\left(D e g_{\text {old }}\right)$ to a new value $\left(D e g_{\text {new }}\right)$ such as:

$$
\text { Avg. Diff } f_{u}^{k}=\operatorname{rand} *\left(\operatorname{Deg}_{n e w_{u}}^{k}-\operatorname{Deg}_{\text {old }}^{k} * T \cdot F_{\text {val }}\right)
$$

4. Update student degree in lecture $u$ using Avg. Diff.:

$$
D e g_{u, z}^{k+1}=D e g_{u, z}^{k}+\operatorname{Avg} \cdot \operatorname{Diff} f_{u}^{k}
$$

5. Learning process, in this stage each student is gain information from their classmates through this process:

- If $\operatorname{Deg}_{u, z}$ is better than $\operatorname{Deg}_{u, b}$ then

$$
D e g_{u, z}^{k+1}=D e g_{u, z}^{k}+\operatorname{rand} *\left(\operatorname{Deg}_{u, b}^{k}-\operatorname{Deg}_{u, z}^{k}\right)
$$

- $\quad$ If $D e g_{u, z}$ worse than $\operatorname{Deg}_{u, b}$ then,

$$
\operatorname{Deg} g_{u, z}^{k+1}=D e g_{u, z}^{k}+\operatorname{rand} *\left(\operatorname{Deg}_{u, z}^{k}-\operatorname{Deg}_{u, b}^{k}\right)
$$

Where, $D e g_{u, z}^{k}, D e g_{u, b}^{k}$ are average degree of lecture $u$ for the students $z \& b$ respectively.

6. Repeat steps (2-5) until convergence criteria confirmed, which is maximum number of iterations.

7. End.

Table 2. Optimal parameters of BTLBO algorithm

\begin{tabular}{ll}
\hline Parameter & Value \\
\hline Pop. $\boldsymbol{N O}$. & 20 \\
Maximum number of iterations & 50 (33 bus) \\
(RDS) & 100 (69 bus) \\
\hline
\end{tabular}

\subsubsection{DDE algorithm}

This a new optimization algorithm discovered by Kaveh and Farhoudi in 2013 that mimic the echo of clicks emits from dolphins, and used for hunting process [26, 27]. The original version of this algorithm has been used previously to solve the power system problems such as the economic dispatch problem and proved its validity and efficiency while the binary version of this algorithm is used with DSR or OCP problems for the first time in this paper. The steps of the DDE algorithm are as follows:

1. Initializing DDE parameters as given in Table 3.

2. Determine Predefined Possibility (P.poss) based on Convergence Factor $\left(C . F_{\cdot 1}\right)$ for current loop $\left(\operatorname{Loop}_{c}\right)$ :

$$
P \cdot \text { poss }_{c}=C \cdot F_{\cdot 1}+\left(1-C . F_{\cdot 1}\right) * \frac{\text { Loop }_{c}-1}{\text { max.Loop.NO.-1 }}
$$

3. Measure Suitability $\left(S_{u}\right)$ for each location $(L)$ where total number of locations is Loc. NO..

4. Define $(K)$ factor bounds that depends on the active Radius(Rac.) as:

$$
K=- \text { Rac. }: \text { Rac. }
$$


5. Determine Incremental Function $(I . F$.$) for each location depend on value of K$ :

- If $|K| \neq R a c$. then,

$$
I . F_{\cdot L}=\frac{1}{R_{a c .}} *\left(R_{a c .}-|K|\right) * S_{u}(L)+I . F_{\cdot L}
$$

- If $|K|=R a c$. which means the superior location is found then,

$$
\text { I. } F_{\cdot L}=0
$$

6. Compute probability of select I.F. for variable $(V r)$ for best location by:

$$
p . \operatorname{sel}_{V r}=\frac{I \cdot F \cdot L V r}{\sum_{V r=1}^{V r \cdot N o \cdot I . F \cdot L V r}}
$$

Where, $p . s_{V r}$, and $V r$. No. are selection probability and number of variables respectively.

7. Divide $p$. sel $_{V r}$ for all incremental for all variables into :

$$
p . \text { sel }_{V r}=\left\{\begin{array}{cc}
\text { P.poss } & \text { for superior location } \\
(1-\text { P.poss }) * p . \text { sel }_{V r} & \text { else }
\end{array}\right.
$$

8. Repeat steps (2-7) until max. Loop. NO. is reached.

9. End.

Table 3. Optimal parameters of DDE algorithm

\begin{tabular}{ll}
\hline Parameter & Value \\
\hline Pop. NO. (Loc. NO.) & 20 \\
Max. Number of iterations (max. Loop. NO.) & $50(33$ bus $)$ \\
& $100(69$ bus $)$ \\
C. $\boldsymbol{F}_{\mathbf{1}}$ & 0.1 \\
Rac. & 2 \\
\hline
\end{tabular}

\section{RESULTS AND ANALYSIS}

The above three algorithms MBBO, BTLBO and DDE are developed using m-file/MATLAB R2018b program. These developed algorithms have been applied for two techniques (DSR only and DSROCP) with two standard IEEE RDS (33 bus and 69 bus). The line and bus data for two test cases are given in reference [28] and the test done for one year using constant power load. The results of these techniques are inserted in tables below for three algorithms.

\subsection{IEEE - 33 bus}

This network (12.66 KV, $100 \mathrm{MVA})$ with load (3715 KW, $2300 \mathrm{KVAr}$ ) and contains (33 buses, 37 branches, 32 closed and 5 opened switches). Figure 1 explains the single line diagram for this system. The results of using two techniques with three optimization algorithms are given in Table 4 and Table 5 respectively. Figure 2 to Figure 5 show the graphs of voltage profile and loss convergence using two techniques with three optimization algorithms respectively.

Also, Table 4 and Table 5 contain comparison with Harmony Search Algorithm (HSA) [29], Cuckoo Search Algorithm (CSA) [30] and Binary Paricle Swarm Optimization (B-PSO) algorithm [31] under the same constraints and objective functions. In addition, these tables also explain better loss reduction and confirming voltage and capacitor size constraints $(2300 \mathrm{KVAr})$ of proposed algorithms while the literature work did not satisfy these constraints. MBBO algorithm perform better loss reduction and fast convergence than other two proposed algorithms and literature works for two techniques and achieve lower capacitor sizes to achieve this reduction. Also, the performance of other proposed algorithms surpass the previous works in loss reduction and keep the RDS constraints. 


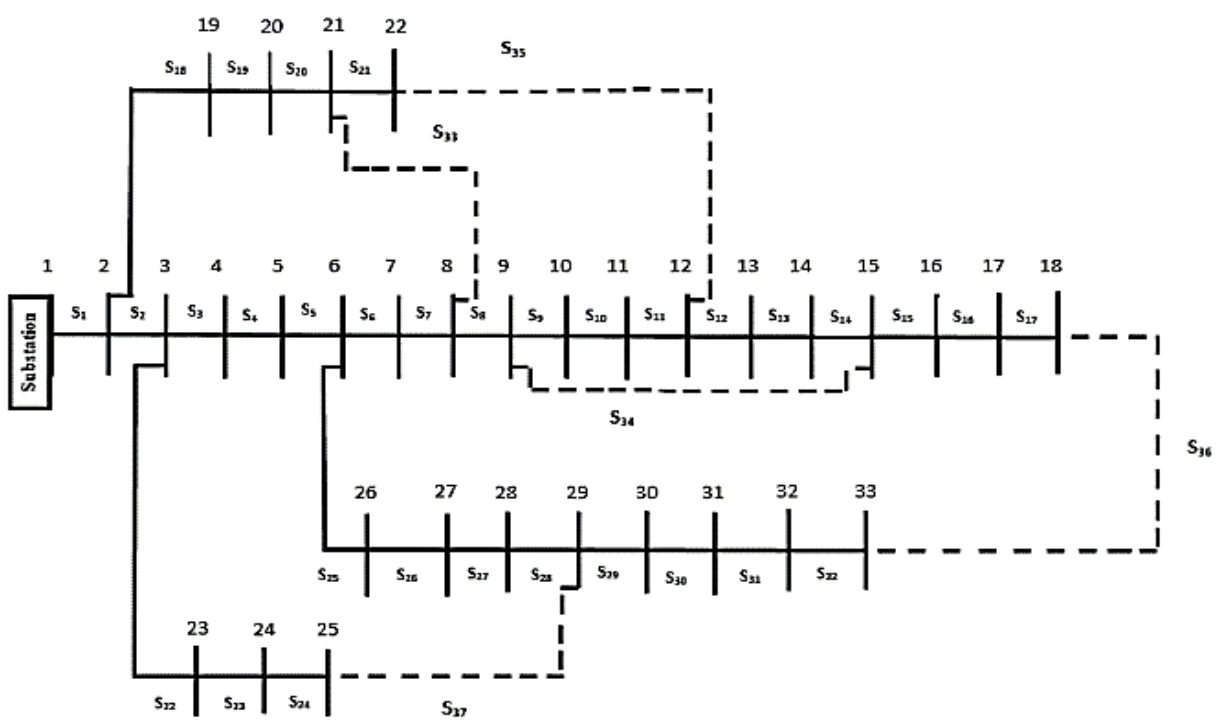

Figure 1. Single line diagram of IEEE- 33 bus

Table 4. Results and comparisons of 33 bus with individual DSR technique

\begin{tabular}{lllll}
\hline Algorithms & Real power losses (KW) & Min bus voltage (p. u.) & Tie switches & \% Loss reduction \\
\hline Base case & 202.6771 & 0.91306 & {$[33,34,35,36,37]$} & - \\
MBBO & 129.9043 & 0.95 & {$[37,33,14,25,11]$} & 35.9058 \\
BTLBO & 135.0425 & 0.95 & {$[28,13,7,8,36]$} & 33.3706 \\
DDE & 135.0425 & 0.95 & {$[36,7,8,13,28]$} & 33.3706 \\
HSA [29] & 137.78 & 0.9301 & {$[33,14,8,32,28]$} & 32.02 \\
CSA [30] & 138.87 & 0.94235 & {$[7,9,14,32,37]$} & 31.482 \\
B-PSO [31] & 141.6346 & 0.9413 & {$[7,11,14,28,32]$} & 30.118 \\
\hline
\end{tabular}

Table 5. Results and comparisons of 33 bus with dual DSR-OCP technique

\begin{tabular}{llllll}
\hline Algorithm & $\begin{array}{l}\text { Real power } \\
\text { losses (KW) }\end{array}$ & $\begin{array}{l}\text { Min. bus } \\
\text { voltage (p. u.) }\end{array}$ & $\begin{array}{l}\text { Capacitor } \\
\text { size (KVAR), (location) }\end{array}$ & Tie switches & $\begin{array}{l}\% \text { Loss } \\
\text { reduction }\end{array}$ \\
\hline Base case & 202.6771 & 0.91306 & - & {$[33,34,35,36,37]$} & - \\
MBBO & 110.6161 & 0.95 & {$[300(12), 300(25), 300(20)]$} & {$[37,33,14,25,11]$} & 45.4225 \\
BTLBO & 115.6684 & 0.9554 & {$[150(13), 300(29), 900(30)]$} & {$[28,13,7,8,36]$} & 42.9297 \\
DDE & 111.8599 & 0.96114 & {$[600(30), 300(33), 900(3)]$} & {$[36,7,8,13,28]$} & 44.8088 \\
HSA [29] & 119.72 & 0.9411 & {$[900(6), 300(28), 600(29)$,} & {$[33,14,8,32,28]$} & 40.93 \\
\hline
\end{tabular}

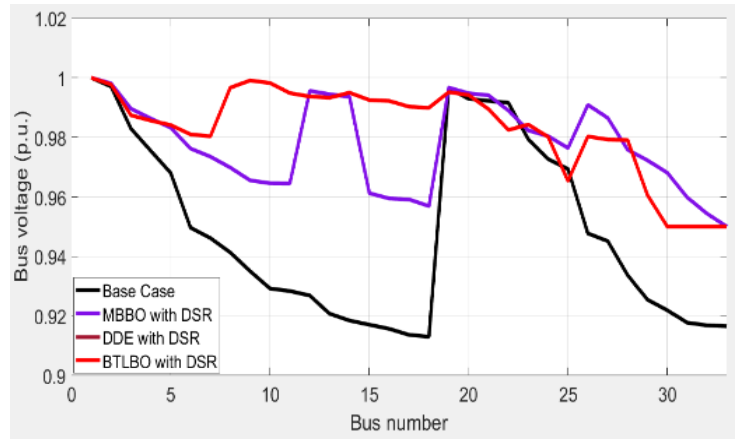

Figure 2. Voltage profile for 33-bus with DSR

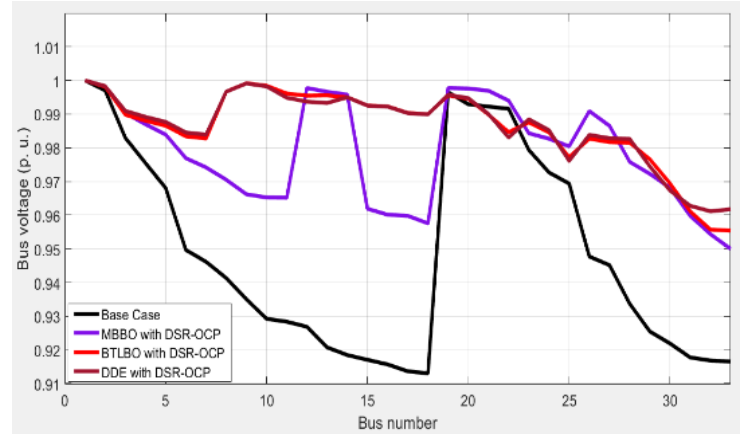

Figure 3. Voltage profile for 33-bus with DSR-OCP 


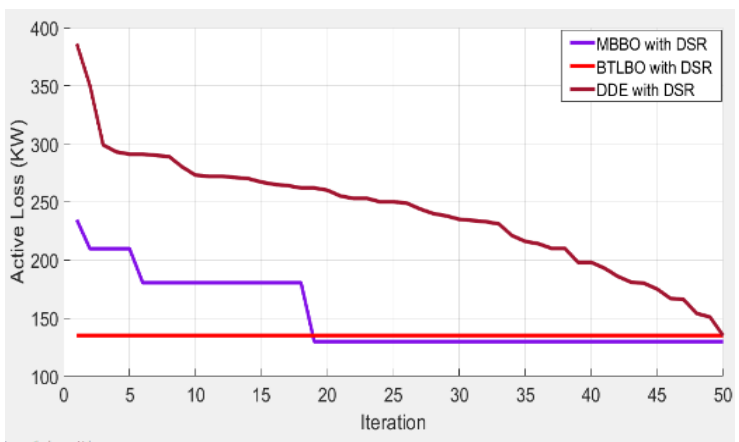

Figure 4. Losses graph for 33-bus with DSR

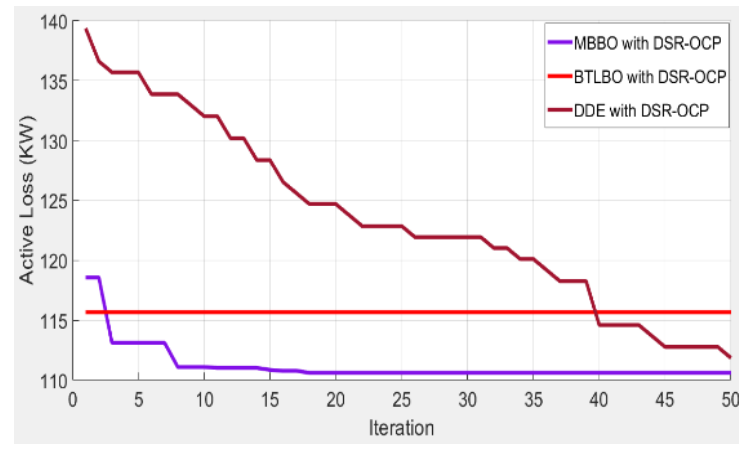

Figure 5. Losses graph for 33-bus with DSR-OCP

\subsection{IEEE - 69 bus}

This network (12.66 KV, 100 MVA) with load (3802 KW, $2695 \mathrm{KVAr}$ ) and contains (69 buses and 68 branches, 68 normally closed and 5 tie switches). Figure 6 explains the single line diagram for this system. The results of using two techniques with three optimization algorithms are given in Table 6 and Table 7 respectively. Figure 7 to Figure 10 show the graphs of voltage profile and loss convergence using two techniques with three optimization algorithms respectively.

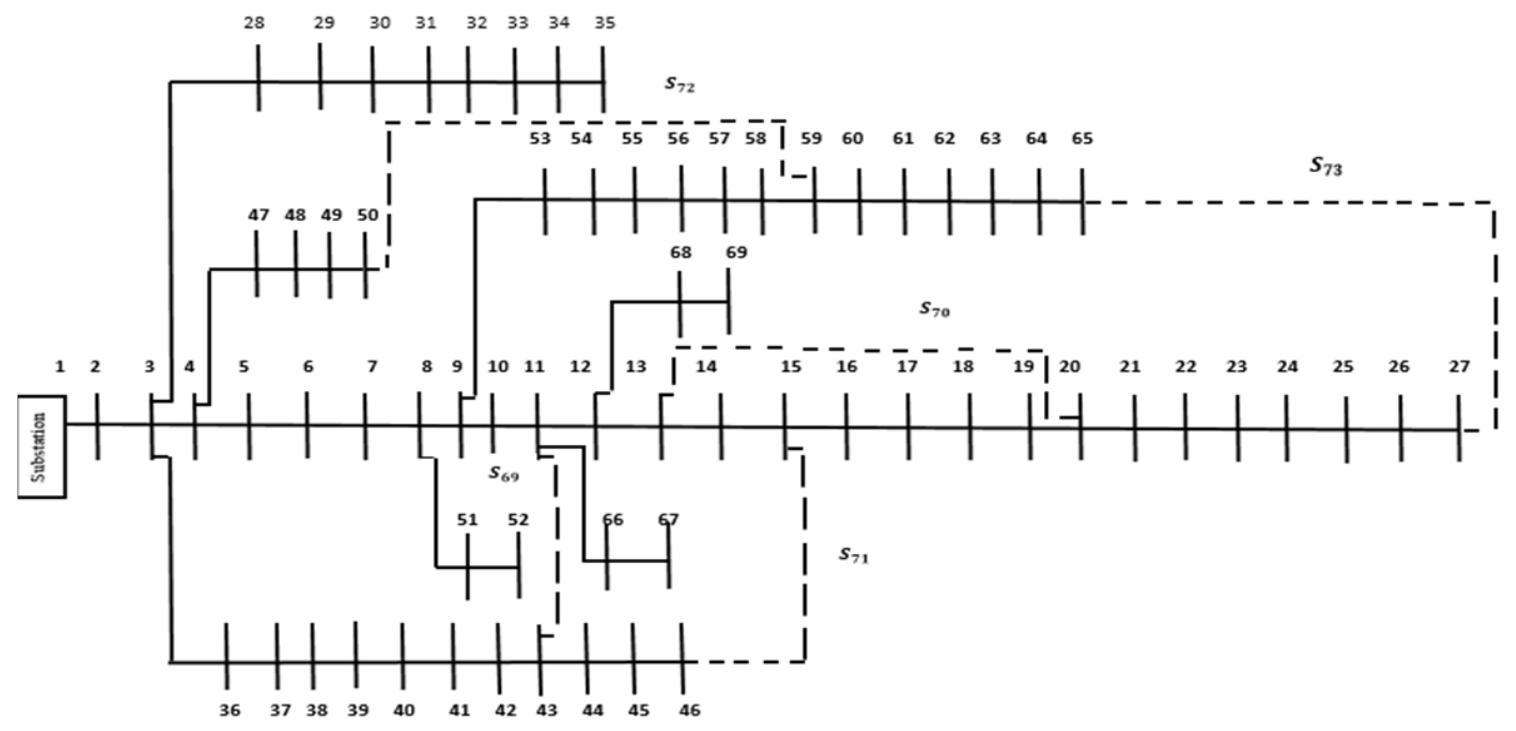

Figure 6. Single line diagram of IEEE- 69 bus

Table 6. Results and comparisons of 69 bus with DSR technique

\begin{tabular}{lllll}
\hline Algorithms & $\begin{array}{l}\text { Real power } \\
\text { losses (KW) }\end{array}$ & $\begin{array}{l}\text { Min bus } \\
\text { voltage (p. u.) }\end{array}$ & Tie switches & $\begin{array}{l}\text { \% Loss } \\
\text { reduction }\end{array}$ \\
\hline Base case & 224.9606 & 0.90901 & {$[69,70,71,72,73]$} & - \\
MBBO & 70.5978 & 0.99048 & {$[14,60,48,12,10]$} & 68.6177 \\
BTLBO & 154.3262 & 0.98856 & {$[12,60,15,6,10]$} & 31.3986 \\
DDE & 119.6588 & 0.98679 & {$[14,53,11,9,60]$} & 46.809 \\
CSA [30] & 98.5680 & 0.9495 & {$[14,57,61,69,70]$} & 56.1843 \\
B-PSO [31] & 98.5952 & 0.9495 & {$[14,58,61,69,70]$} & 56.1722 \\
CBGA [32] & 99.62 & NK $^{*}$ & {$[59,70,71,15,61]$} & 55.716 \\
\hline NK $:$ Not Known & & &
\end{tabular}


Table 7. Results and comparisons of 69 bus with DSR-OCP technique

\begin{tabular}{llllll}
\hline Algorithms & $\begin{array}{l}\text { Real power } \\
\text { losses (KW) }\end{array}$ & $\begin{array}{l}\text { Min bus voltage } \\
\text { (p. u.) }\end{array}$ & $\begin{array}{l}\text { Capacitor } \\
\text { size }(\text { KVAR), (location) }\end{array}$ & Tie switches & $\begin{array}{l}\% \text { Loss } \\
\text { reduction }\end{array}$ \\
\hline Base case & 224.9606 & 0.90901 & - & {$[69,70,71,72,73]$} & - \\
MBBO & 58.6166 & 0.9911 & {$[300(21), 300(50), 300(11)]$} & {$[14,60,48,12,10]$} & 73.9436 \\
BTLBO & 116.6786 & 0.99001 & {$[300(25), 300(37), 900(49)]$} & {$[12,60,15,6,10]$} & 48.1338 \\
DDE & 105.554 & 0.98744 & {$[600(48), 600(6), 300(68)]$} & {$[14,53,11,9,60]$} & 53.0789 \\
CBGA [32] & 68.50 & NK & {$[300(62), 300(63)]$} & {$[56,70,15,71,62]$} & 69.5502 \\
\hline
\end{tabular}

In addition, Table 6 and Table 7 contains comparison with CSA [30], B-PSO algorithm [31], Chu and Beasley based Genetic Algorithm (CBGA) [32] under the same constraints and objective functions. Also, from these tables the MBBO algorithm explained its significance reduction of losses over than the other algorithms in literature works and confirmed all the operational and technical constraints for both techniques. The literature works satisfied some of progress in real power losses reduction but did not maintain the voltage limits, while the DDE and BTLBO algorithms in the current work has been working to reduce real losses while maintaining voltage constraints.

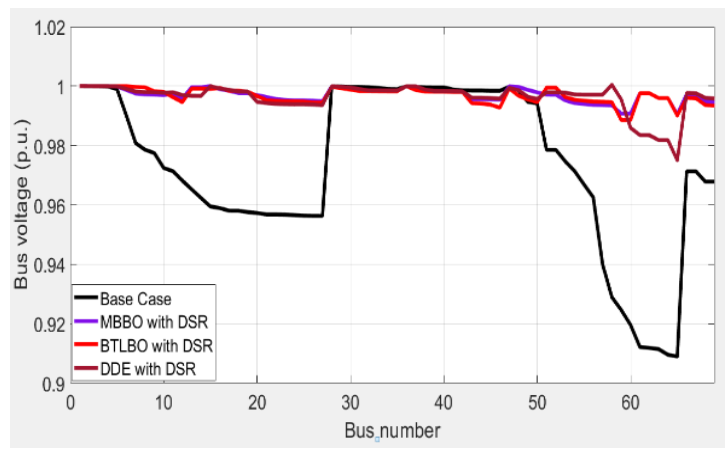

Figure 7. Voltage profile for 69-bus with DSR

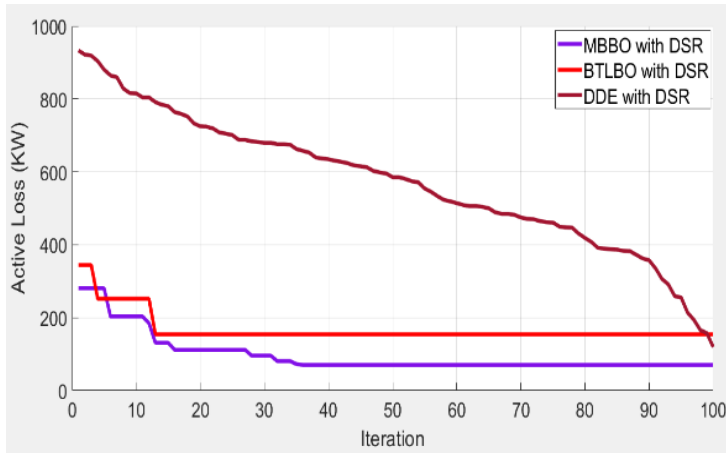

Figure 9. Losses graph for 69-bus with DSR

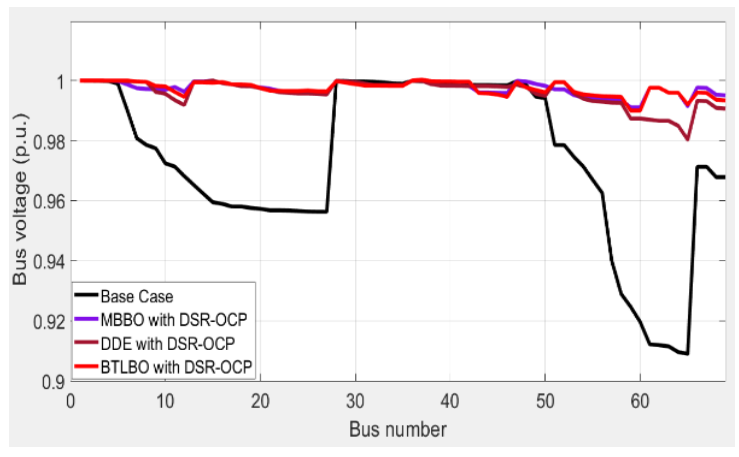

Figure 8. Voltage profile for 69-bus with DSR-OCP

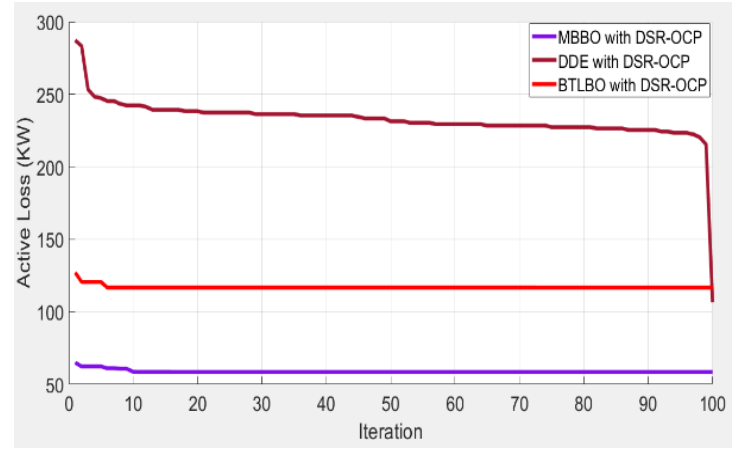

Figure 10. Losses graph for 69-bus with DSR-OCP

It is quite clear from the results of tables and figures for two test systems the individual and dual techniques greatly improve the system in terms of real power loss reduction and voltage profile improvement, but the dual technique provided the power systems with better characteristic over the individual technique. The results also showed the effectiveness of the MBBO algorithm to provide a stable, fast and superior performance in comparison with other algorithms in individual and dual techniques for both loss reduction and voltage profile improvement. Moreover, the DDE algorithm provides better performance over the BTLBO algorithm in both techniques and two case studies. In addition, the modified versions of these algorithms provided a better performance over their original algorithms for the proposed techniques through avoiding the local minimum solution. Finally, the results that obtained based on these algorithms for locations of capacitors lead to further loss reduction over the conventional methods in the literature. 


\section{CONCLUSION}

DSR and OCP are the most efficient and economic techniques for better performance of the RDS. Three different modified and enhanced optimization algorithms have been used to search the optimal topology that achieves minimum losses and improved voltage profile. Comparisons are made among them using different networks and techniques. In this paper, two techniques used, first, the DSR technique applied individually; second the dual technique of DSR-OCP. These techniques applied tested on the two networks with different sizes and topologies to test the efficiency of proposed algorithms and techniques. A DBFSM load flow method implemented for calculation of bus voltage, branch current and total losses to obtain robust results better than those obtained using conventional load flow methods. The results in two power systems show that the MBBO algorithm has better performance when compared to the other mentioned algorithms for different techniques and networks, and as expected, a dual approach of both techniques is better than use it individually. A previous works implemented to make a comparison with the proposed algorithms and the difference between different algorithms will be bigger and clear when use different techniques and networks. The comparison results demonstrated that the proposed algorithms keep the standard RDS constraints while the literature works have been broken it. In addition, the technique of locations for capacitors provided a further improvement in RDS performance over the LSI based approachs for a large scale RDS. Finally, the results proved the effectiveness, robustness and steadiness of these modified algorithms so can utilized for other optimization applications in the power system.

\section{REFERENCES}

[1] S. M. Banteywalu and C. Renald, "Distribution system loss reduction in addisababa north substation," International Conference on Innovations in Power and Advanced Computing Technologies [i-PACT2017], pp.1-8, 2017.

[2] A. Merlin and H. Back, "Search for a minimal-loss operating spanning tree configuration in an urban power distribution system," In Proc. 5th Power System Computation Conference (PSCC), Cambridge, UK, pp. 1-18, 1975.

[3] H. K. Verma and P. Singh, "Optimal reconfiguration of distribution network using modified culture algorithm," Journal of The Institution of Engineers (India): Series B, vol. 99(6), pp. 613-622, 2018.

[4] K. Sandhya and D. S. K. Kanth, "Active power loss minimization in radial distribution system using network reconfiguration in the precence of distributed generation," International Journal of Advances in Electronics and Computer Science, vol. 2(6), pp. 89- 95, 2015.

[5] M. A. Heidari, "Optimal network reconfiguration in distribution system for loss reduction and voltage-profile improvement using hybrid algorithm of PSO and ACO," 24th International Conference \& Exhibition on Electricity Distribution(CIRED), pp. 2458-2461, 2017.

[6] R. Chidanandappa, et al.,"Genetic algorithm based network reconfiguration in distribution systems with multiple DGs for time varying loads," Procedia Technology 21 (2015), pp. 460-467, 2015.

[7] P. Kumar and Somvir, "Network reconfiguration of distribution system using particle swarm optimization," International Journal Of Engineering And Computer Science (IJECE), vol. 5(8), pp. 17369-17374, 2016.

[8] M. Sedighizadeh, et al.,"Reconfiguration of radial distribution systems with fuzzy multi-objective approach using modified big bang-big crunch algorithm," Arabian Journal for Science and Engineering, vol. 39(8), pp. 6287-6296, 2014.

[9] M. E. Baran and F. F. Wu,"Optimal capacitor placement on radial distribution systems," IEEE Transactions on Power Delivery, vol. 4(1), 1989.

[10] A. A. Abou El-Ela, et al.,"Optimal capacitor placement in distribution systems for power loss reduction and voltage profile improvement," The Institution of Engineering and Technology, vol. 10(5), pp. 1209-1221, 2016.

[11] R. Sirjani and M. G. Bade "A global harmony search algorithm for finding optimal capacitor location and size in distribution networks," Journal of Central South University, vol. 22(5), pp. 1748-1761, 2015.

[12] S. Bhongade and S. Arya, "Optimal capacitors in radial distribution system for loss reduction and voltage enhancement," Indonesian Journal of Electrical Engineering and Computer Science, vol. 2(3), pp. 566-582, 2016.

[13] Y. M. Shuaib, et al.,"Optimal capacitor placement in radial distribution system using gravitational search algorithm," International Journal of Electrical Power \& Energy Systems, vol. 64, pp. 384-397, 2015.

[14] D. B. Prakash, C. Lakshminarayana, "Optimal siting of capacitors in radial distribution network using whale optimization algorithm," Alexandria Engineering Journal, vol. 56(4), pp. 499-509, 2017.

[15] Radhika Priyadarshini et al.," Joint optimization for capacitor placement and network reconfiguration for loss reduction in distribution system", 2014 IEEE International Conference on Power Electronics, Drives and Energy Systems (PEDES), Mumbai, India, 2014.

[16] Leonardo W. de Oliveira, et al., "Optimal reconfiguration and capacitor allocation in radial distribution systems for energy losses minimization," International Journal of Electrical Power \& Energy Systems, vol. 32(8), pp. 840-848, 2010.

[17] N. Kumar, M. Ramraj, "Combined reconfiguration and capacitor placement for distribution system volt/var control through opposition based differential evolution algorithm," Journal for Control, Measurement, Electronics, Computing and Communications, vol. 56(2), pp. 140-148, 2015.

[18] J. Teng,"A direct approach for distribution system load flow solutions," IEEE Transactions on Power Delivery, vol. 18(3), pp. 882-887, 2003 
[19] M. F. Sulaima, et al., "A $16 \mathrm{kV}$ distribution network reconfiguration by using evolutionaring programming for loss minimizing," International Journal of Applied Engineering Research, vol. 9(10), pp. 1223-1238, 2014.

[20] M. M. Aman, et al., "Optimum network reconfiguration based on maximization of system loadability using continuation power flow theorem," International Journal of Electrical Power and Energy Systems, vol. 54, pp. 123-133, 2014.

[21] Dan Simon, "Biogeography-based Optimization," IEEE Trans. On Evolutionary Computation, vol. 12(6), pp. 702-713, Dec. 2008.

[22] A. R. Al-Roomi, et al.,"Essential modifications on biogeography-based optimization algorithm," Second International Conference on Advanced Information Technologies and Applications (ICAITA-2013), vol. 3(8), Dubai, UAE, pp. 141-160, 2013.

[23] O. B. Haddad, et al.,"Biogeography-based optimization algorithm for optimal operation of reservoir systems," Journal of Water Resources Planning and Management, vol. 142(1), 2016.

[24] R.V. Rao, et al., "Teaching-learning based optimization algorithm for unconstrained and constrained real parameter optimization problems," Engineering optimization, vol 44 (12), pp.1447-1462, 2012.

[25] Z. Yang, et al., "Binary teaching-learning based optimization for power system unit commitment," UKACC 11th International Conference on Control (CONTROL), Belfast, UK, pp. 1-6, 2016.

[26] A. Kaveh and N. Farhoudi, "A new optimization method: dolphin echolocation," Advances in Engineering Software, vol. 59, pp. 53-70, 2013.

[27] K. Lenin, et al., "Dolphin echolocation algorithm for solving optimal reactive power dispatch problem," International Journal of Computer (IJC), vol. 12(1), pp. 1-15, 2014.

[28] S. Naveen, et al.,"Distribution system reconfiguration for loss minimization using modified bacterial foraging optimization algorithm," International Journal of Electrical Power and Energy Systems, vol. 69, pp. 90-97, 2015.

[29] R. S. Rao," An hybrid approach for loss reduction in distribution systems using harmony search algorithm," International Journal of Electrical and Computer Engineering, vol. 4(3), pp.557-563, 2009.

[30] T. T. Nguyen and A. V.Truong," Distribution network reconfiguration for power loss minimization and voltage profile improvement using cuckoo search algorithm," International Journal of Electrical Power \& Energy Systems, vol. 68 , pp. 233-242, 2015.

[31] G. S. Shehu, "Distribution network reconfiguration for loss reduction and voltage profile improvement using BPSO," 3th International Conference, ICAT'16, International Conference on Advanced Technology \& Sciences, Konya, Turkey, vol. 3, 2016.

[32] M. A. N. Guimaraes and C. A. Castro, "An efficient method for distribution systems reconfiguration and capacitor placement using a Chu-Beasley based genetic algorithm," 2011 IEEE Trondheim PowerTech, Trondheim, Norway, pp. 1-7, 2011.

\section{BIOGRAPHIES OF AUTHORS}
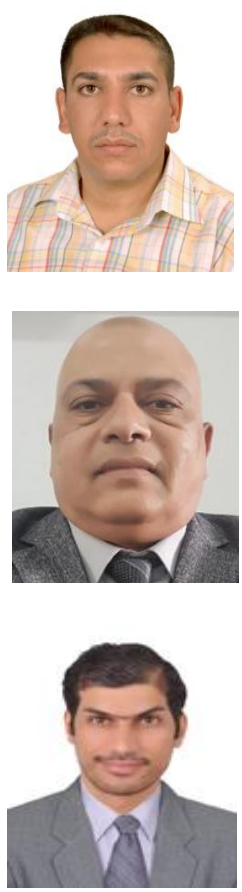

Ali Nasser Hussain was born in Iraq on April 30, 1974. He received his B.Sc. and M.Sc. in Electrical \& Electronics Engineering, University of Technology, Baghdad, Iraq, in 1998 and in 2005 respectively and his PhD degrees in Electrical Engineering from University Malaysia Perlis (UniMAP), Perlis, Malaysia in 2014. Since 2004 he is a senior lecturer in the Electrical Engineering Technical College at Middle Technical University. His current research interests include power system operation and control, electrical power system stability and intelligent optimization, renewable energy, robust control.

Waleed Khalid Shakir Al-Jubori was born in Babil,Iraq in 1964. He received his B.Sc degree from University of Baghdad, College of Engineering in 1987. He received his M.Sc degree from University of Technology in 2005 and his Ph.d degree from University of Technology in 2017. $\mathrm{He}$ is a teacher in Department of Electrical Power Engineering Techniques, Al-Mussaib Technical College, AL-Furat AL-Awsat Technical University in Iraq. His areas of interest include power system stability and control, FACTS devices, and application of artificial intelligent algorithms in power system analysis.

Haider Fathi Kadom was born in Iraq on November 6, 1986. He received his B.Sc. in Electrical power Techniques Engineering, AL-Furat Al-Awsat Technical University, Babil, Iraq, in 2008. He is currently pursuing his M.Sc. program in electrical technical Engineering with emphasis in Distribution Systems. His current research interests include operation and control of power system, electrical power system stability and intelligent optimization. 